\title{
PARALLEL PROCESSING OF BIG POINT CLOUDS USING Z-ORDER-BASED PARTITIONING
}

\author{
C. Alis*, J. Boehm, K. Liu
} Dept. of Civil, Environmental and Geomatic Engineering, University College London, Gower Street, London, UK
c.alis@ucl.ac.uk

Commission II, WG II/2

KEY WORDS: Z-order, Spatial partitioning, Big Data, LiDAR, Point Cloud, Apache Spark

\begin{abstract}
:
As laser scanning technology improves and costs are coming down, the amount of point cloud data being generated can be prohibitively difficult and expensive to process on a single machine. This data explosion is not only limited to point cloud data. Voluminous amounts of high-dimensionality and quickly accumulating data, collectively known as Big Data, such as those generated by social media, Internet of Things devices and commercial transactions, are becoming more prevalent as well. New computing paradigms and frameworks are being developed to efficiently handle the processing of Big Data, many of which utilize a compute cluster composed of several commodity grade machines to process chunks of data in parallel.

A central concept in many of these frameworks is data locality. By its nature, Big Data is large enough that the entire dataset would not fit on the memory and hard drives of a single node hence replicating the entire dataset to each worker node is impractical. The data must then be partitioned across worker nodes in a manner that minimises data transfer across the network. This is a challenge for point cloud data because there exist different ways to partition data and they may require data transfer.

We propose a partitioning based on $Z$-order which is a form of locality-sensitive hashing. The $Z$-order or Morton code is computed by dividing each dimension to form a grid then interleaving the binary representation of each dimension. For example, the Z-order code for the grid square with coordinates $\left(x=1=01_{2}, y=3=11_{2}\right)$ is $1011_{2}=11$. The number of points in each partition is controlled by the number of bits per dimension: the more bits, the fewer the points. The number of bits per dimension also controls the level of detail with more bits yielding finer partitioning. We present this partitioning method by implementing it on Apache Spark and investigating how different parameters affect the accuracy and running time of the $k$ nearest neighbour algorithm for a hemispherical and a triangular wave point cloud.
\end{abstract}

\section{INTRODUCTION}

The release of big point cloud data sets e.g., the Actueel Hoogtebestand Nederland 2 (AHN2) (Swart, 2010) dataset, has enabled researchers to have access to a high-quality and high-density point cloud of a large area. It has also brought to light the challenges of dealing with point clouds with a total disk size comparable to the volume of what can be considered as Big Data. AHN2, for example, consists of 446 billion points spread across 1351 LAS zip files for a total uncompressed size of 9 TB. Handling and processing the data on a single machine is challenging even impractical.

The problem of handling large amounts of data is not unique to those working with big point clouds. In recent years, with the rise of new communication and information technologies as well as with improved access to affordable computing power, substantial work is being done in developing systems, frameworks and algorithms for handling voluminous amounts of high-dimensionality and quickly accumulating data, collectively known as Big Data.

By definition, Big Data is voluminous that it typically does not fit on a single hard drive. Even if it does, transferring data from storage to processing machine over a slow bus (network) results in a huge performance penalty. The solution to these problems would be to split the data into different partitions which may be stored across several processing machines. We want the data to be on the processing machine as much as possible, a concept known as data locality, to avoid slow network transfers.

\footnotetext{
${ }^{*}$ Corresponding author
}

Individual data points may be randomly assigned to different partitions to ensure parallel operations are balanced across processing machines. However, several operations on point clouds require neighbourhoods of points as input. Thus, a method of partitioning a point cloud is required such that points that are geometrically close are assigned into the same partition.

One of the systems developed for Big Data processing is Apache Spark ${ }^{1}$. It can handle data stored across multiple partitions and user code can be written in Java, Scala, Python and R. Third party dynamically linked libraries can also be called using the facilities provided by the programming language of the user code. Significant time and effort can then be saved by adapting and using previously written code and compiled point cloud libraries.

In this paper, we present a partitioning method based on the $Z$ order. We show that nearest-neighbours-based, or focal, operations can be performed by processing each partition independently and that doing so is a quick and precise approximation. We also show how a third party dynamically linked library can be used to process point clouds on Apache Spark.

We propose indexing the points with a space-filling curve, in particular, a $Z$-order curve, which would serve as the basis for partitioning. It is a form of a locality-sensitive hashing. $Z$-order curves have been in use in grouping and indexing multidimensional points (Orenstein and Manola, 1988, Lawder and King, 2000). More recently, $Z$-order curves are being employed in constructing nearest neighbour graphs in parallel on a single machine (Connor and Kumar, 2010, Orenstein and Manola, 1988),

\footnotetext{
${ }^{1}$ spark. apache.org
} 


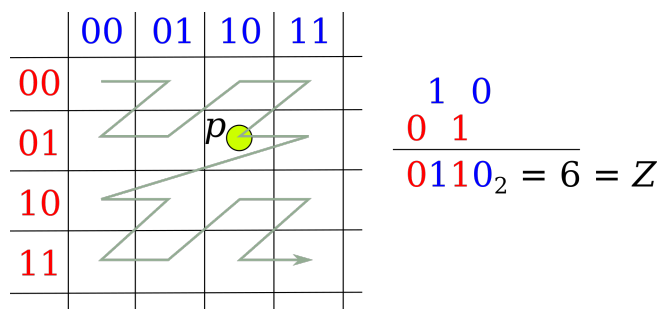

Figure 1: 2D $Z$-order

indexing spatial data on distributed databases (Nishimura et al., 2012, Wei et al., 2014, Lawder and King, 2000, Connor and Kumar, 2010), and partitioning spatial data on MapReduce (Eldawy et al., 2015, Nishimura et al., 2012).

The $Z$-order, also known as Morton code, of a point $p$ is assigned as follows. The bounding box is first divided into $2^{b}$ partitions per dimension (see Figure 1 for an illustration of a 2D case) and are then sequentially assigned a $b$-bit number. The number of the partition where $p$ is found for each dimension is then interleaved and this becomes the $Z$-order code.

In the illustration, two bits $(b=2)$ were assigned per dimension hence the result is a grid of $2^{2}$ partitions $\times 2^{2}$ partitions. The index of the lower leftmost partition is $0000_{2}=0$ whilst that of the upper rightmost partition is $1111_{2}=15$. The point $p$ is in coordinates $\left(10_{2}, 01_{2}\right)$. Interleaving the coordinates, we get $0110_{2}=6$ and this is the $Z$-order. Connecting the centre of each partition sequentially based on the index would result to a $Z$-like curve hence the name $Z$-order.

We implement this partitioning method in Apache Spark which is a system for distributed computing. It can scale from a single machine to hundreds of thousands of worker machines. It can also read from various data stores such as from a local file or from a distributed file system such as the Hadoop Distributed File System (HDFS). User code can be written in Java, Scala, Python and R. It is an open source project under very active development and with a vibrant user community.

The core abstraction of Spark is the resilient distributed dataset (RDD), which is a distributed collection of elements. Instead of immediately executing an operation on an RDD, Spark instead builds an execution graph until it encounters an operation, known as an action, to force the graph to be executed. This allows the actual execution to be optimized based on succeeding operations. For example, instead of reading an entire file, Spark may read only those parts that will be processed in succeeding steps.

Spark has also introduced the DataFrames application programming interface (API), which essentially wraps an RDD into SQLlike tables. This API also eases optimization by adding more context to the desired action. Another benefit of the API is that the methods are implemented in Java and Scala, the native languages of Spark, regardless of the language used by the user. This is a major boost for Python and $\mathrm{R}$ users, which are typically executed more slowly than Java or Scala.

Logically, a Spark system can be divided into driver and executors. The driver is the machine process that reads the input user code, creates the execution graph and breaks it down into stages, which are chunks of parallelizable subgraphs in the execution graph. The stages are then distributed among the executors, which are typically several, and may or may not reside on the same machine as the driver. Parallelization is implemented by having many executors, each working on a stage assigned to them.
Datasets are typically composed of several partitions, which may be physically stored on different machines. Executors work on a single partition of data. Before an executor can process a partition, the data in that partition must be located on the same machine as the executor. The process of transferring data from where it is located to where it should be is known as a shuffle. Because it involves reading and writing data from/to disks and/or memory and may pass through the network, it is a slow process and is avoided as much as possible. Unlike Hadoop ${ }^{2}$, however, Spark can store intermediate data in memory rather on disk only.

User scripts written in Java or Scala are executed natively. The data processing and handling of Python scripts are handled by a separate function process but uses the generic distributed computing capabilities in Spark. As mentioned above, DataFrame methods are implemented in Java and Scala. Dynamically linked libraries can be called using the facilities provided by the programming language used by the user.

There is no native support for point clouds in Spark but there are libraries and systems that add support for raster and vector data. SpatialSpark (You et al., 2015) implements spatial join and spatial query for vector data in Spark and may use an R-tree index to speed up point-in-polygon processing. Magellan ${ }^{3}$ is another library for geospatial analytics of vector data using Spark and aims to support geometrical queries and operations efficiently. Geotrellis ${ }^{4}$ is a complete system, not just a library, for geographic data processing based on Spark. It is initially intended for raster data but some support for vector data is also available. It can also employ $Z$-order and Hilbert curve indices.

We propose and explore the accuracy and running time of a handful of workflows which employ $Z$-order-based partitioning to perform nearest-neighbour based computations. We are not aware of any $k$ nearest neighbours $(\mathrm{kNN})$ selection implementation in Spark, however, there are at least two algorithms for $\mathrm{kNN}$ join using MapReduce, which is a framework for parallel processing wherein operations are through a series of map and reduce functions.

The H-zKNNJ algorithm (Zhang et al., 2012) partitions a dataset based on $Z$-order. Multiple copies of each point are created by adding a constant random vector for each copy. Each point is then assigned into partitions based on its and its copies' $Z$-orders. The points in each partition serve as the search space for candidate nearest neighbours of each point in the partition. The candidate nearest neighbours are then grouped by points and reduced to the final $k$ nearest neighbours.

The method proposed by Lu et al. (Lu et al., 2012) partitions the dataset into a Voronoi lattice. It requires the pivot points to be known beforehand. The first MapReduce operation assigns each point into one or more partitions based on different approaches e.g., distance, random or $k$-means. The rest of the method is similar to H-zKNNJ: the partitions act as the search space for candidate nearest neighbours which are then reduced to the final $k$ nearest neighbours.

\section{WORKFLOWS}

We investigate four workflows for performing nearest-neighbour based computations of a big point cloud and were implemented in Apache Spark using Python. To do so, we have to select the $k$ nearest neighbours of each point, which we define as

\footnotetext{
${ }^{2}$ hadoop.apache.org

${ }^{3}$ github.com/harsha2010/magellan

${ }^{4}$ geotrellis.io
} 
Definition 1. ( $k$ nearest neighbors) Given point $p \in S$, point cloud $S$ and an integer $k$, the $k$ nearest neighbors of $p$ in $S$, denoted as $K N N(p)$ is a set of $k$ points in $S$ such that $\forall q \in$ $K N N(p), \forall r \in S \backslash K N N(p),\|p, q\| \leq\|p, r\|$.

Note that $\forall r \in S,\|p, p\|=0 \leq\|p, r\|$, thus, $p \in K N N(p)$. We use the 2D Euclidean distance as the norm.

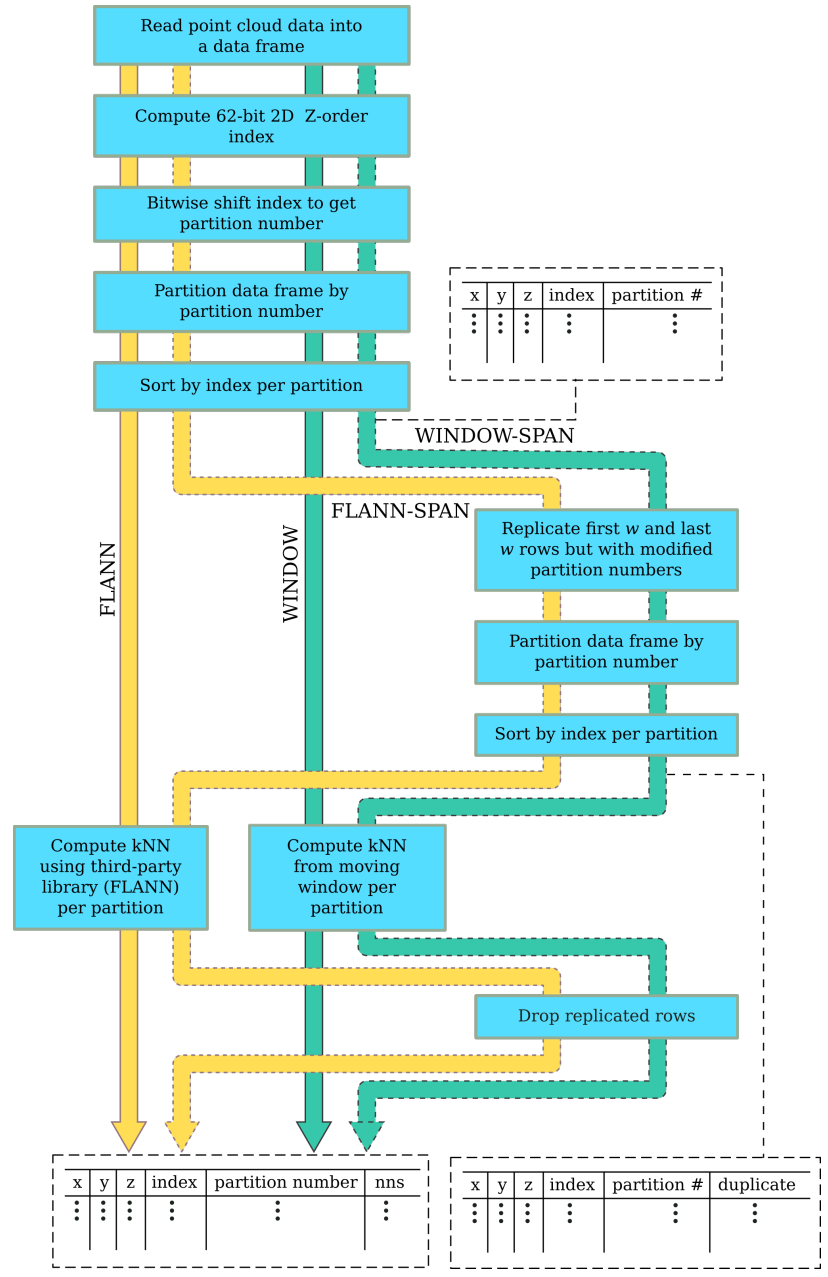

Figure 2: Flowchart of the workflows. The tables surrounded by broken lines show the minimum columns in the data frame at that point in the workflow.

For all workflows (Figure 2), the input point cloud is first converted into a Spark DataFrame, with each point represented as a row. A 62-bit $Z$-order index from the normalised $x$ and $y$ coordinates is then assigned to each point. Its normalisation is based on the bounding box of the entire point cloud with $(0,0)$ being the lower left corner and $\left(2^{31}-1,2^{31}-1\right)$ being the upper right corner. From the index, the $2 b$-bit partition number $(b$ bits per dimension) for each point is computed by bitwise shifting the index to the right by $(62-2 b)$ bits. The dataset is then partitioned by the partition number then sorted by the index within each partition. The call to the partition() method of the data frame would trigger a shuffle.

The first workflow, FLANN, selects the $k$ nearest neighbours $(\mathrm{kNN})$ of each point by passing the contents of each partition to an appropriate third party library, which is Fast Library for Approximate Nearest Neighbors (FLANN) ${ }^{5}$ in our implementation and experiments.

\footnotetext{
${ }^{5}$ www.cs.ubc.ca/research/flann
}

The second workflow, WINDOw, exploits the windowing feature of the Spark DataFrames API. In this workflow, $w$ preceding rows, $w$ succeeding rows and the current row, for a total of at most $(2 w+1)$ rows, are considered in each window. The distance from each neighbouring point to the point is computed and the $k$ points with the smallest distances are then selected as the $k$ nearest neighbours. Neighbourhood-based computations, which may be through a third party library, can then be carried out further on this window.

Points near the partition boundaries may not have access to their true nearest neighbours which may be in other partitions. Partitions can be made to span other partitions by creating a moving window buffer by replicating the first $w$ and the last $w$ rows by as many partitions as needed. After partitioning and sorting with partitions, the result is a data frame of points which can then be treated as the input for FLANN or WINDOW. We call these modified workflows as FLANN-SPAN and WINDOW-SPAN, respectively.

The spanning step is similar to the replication step in the $\mathrm{kNN}$ join algorithms discussed above. However, instead of adding a random vector, as in $\mathrm{H}-\mathrm{zKNNJ}$, or using a heuristic as in the method proposed by $\mathrm{Lu}$ et al. (Lu et al., 2012), we only base the partitioning of the duplicates on the position of the row on the data frame sorted by the 62 -bit $Z$-order.

Note that enabling spanning triggers a shuffle. To summarise, FLANN and WINDOW require at least one shuffle whilst FLANNSPAN and WINDOW-SPAN require at least two shuffles. We expect that a workflow with spanning is slower than one without. With a handicap on runtime, we then have to investigate by how much accuracy improves with spanning, if it does, and what is the performance penalty for that improvement, assuming accuracy does improve.

\section{EXPERIMENTAL RESULTS}

We investigate the accuracy $a$ defined as

$$
a=\frac{n_{\mathrm{c}}}{k}
$$

where $n_{\mathrm{c}}$ is the number of correctly selected points and $k$ is the number of nearest neighbours, of the different workflows. We also look at the running time of the different workflows as various parameters of the point cloud and workflows vary.

We consider two kinds of synthetic point clouds: hemispherical and triangular wave. The former is a smooth point cloud while the latter is a sharper point cloud. The hemisphere is parameterized by its radius $r$ and is in the $+z$ half-space. The triangular wave is parameterized by the number of peaks $p$ and is also in the $+z$ half-space. The wave is travelling along the $x$-axis, that is, $z$ is a function of $x$ only.

The normals of both point clouds point upwards. Thus, the computed normals $\vec{n}_{\mathrm{c}}$ in the workflow are reversed $\left(-\vec{n}_{\mathrm{c}}\right)$ if they are pointing upwards.

All experiments were implemented in Apache Spark 1.6.1 with a local master and 8 executor cores. The memory allocation is $8 \mathrm{~GB}$ for the driver and $1 \mathrm{~GB}$ for the executors. The workflows were implemented in Python.

In these experiments, we measure the performance of the workflows in kNN computations where we used the FLANN library for the FLANN and FLANN-SPAN workflows. 


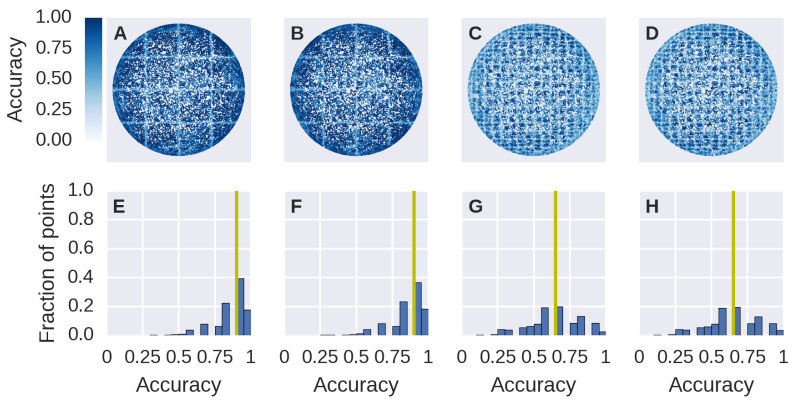

Figure 3: kNN accuracy in processing a hemispherical point cloud for a particular set of parameters for different workflows. In A-D, the points are projected onto the $x-y$ plane. The colour corresponds to the kNN accuracy, defined as the fraction of correctly selected $k$ nearest neighbours, at each point. In E-H, the distribution of the accuracy is shown with the yellow vertical line indicating the value of the median. The workflows are: (A,E) FLANN, (B,F) FLANN-SPAN, $(\mathrm{C}, \mathrm{G})$ WINDOW, $(\mathrm{D}, \mathrm{H})$ WINDOWSPAN. The values of the parameters are bit per dimension $b=2$, number of points per partition $N_{\mathrm{p}}=1000$, radius $r=1$, number of nearest neighbours $k=20$ and look-back/look-ahead length $w=20$.

\subsection{Hemispherical Point Cloud}

The hemispherical point cloud that we tested has a radius $r$, centred at the origin and is in the $+z$ half-space. The motivation of selecting this geometry is to test the workflows on a smooth point cloud.

3.1.1 Spatial Accuracy Figure 3 shows the accuracy for hemispherical point clouds and workflows with bits per dimension $b=2$, number of points per partition $N_{\mathrm{p}}=1000$, radius $r=1$, $k=20$, and window look-back/look-ahead length $w=20$.

For this set of parameters, the results for the spanning and nonspanning versions of FLANN and WINDOW are the same. Gridlike lines can be made out from Figures $3 \mathrm{~A}$ and $3 \mathrm{~B}$ and this is due to the lower accuracy of points near partition boundaries. Gridlike lines are also visible in Figures $3 \mathrm{C}$ and $3 \mathrm{D}$ but there are more lines and the spacing is much less, which suggests that the grid more likely due to $Z$-order indexing rather than due to partition boundaries.

The colours of Figures 3A and 3B are darker than those of Figures $3 \mathrm{C}$ and $3 \mathrm{D}$ suggesting that the former are more accurate than the latter. There is a prominent peak at $a=0.9$ in Figures $3 \mathrm{E}$ and $3 \mathrm{~F}$ corresponding to at least $50 \%$ of the points having an accuracy of at least 0.9. On the other hand, the distribution in Figures 3G and $3 \mathrm{H}$ are more spread and there is a less prominent peak at $a=0.65$. Comparing the median of 0.9 for FLANN and FLANNSPAN with the median of 0.65 for WINDOW and WINDOW-SPAN, we conclude that FLANN and FLANN-SPAN are more accurate than WINDOW and WINDOW-SPAN at least for this set of parameters. Furthermore, WINDOW and WINDOW-SPAN have a minimum accuracy of 0.05 i.e., only the point itself was selected as a nearest neighbour, which is less compared to a minimum accuracy of 0.1 for FLANN and 0.2 for FLANN-WINDOW.

3.1.2 Effect of Different Parameters on Accuracy We now look at the accuracy of the workflows for different parameter values as shown in Figure 4. Except for a few particular instances, the lines are constant with respect to the radius which suggests that accuracy does not depend on the curvature of the point cloud.

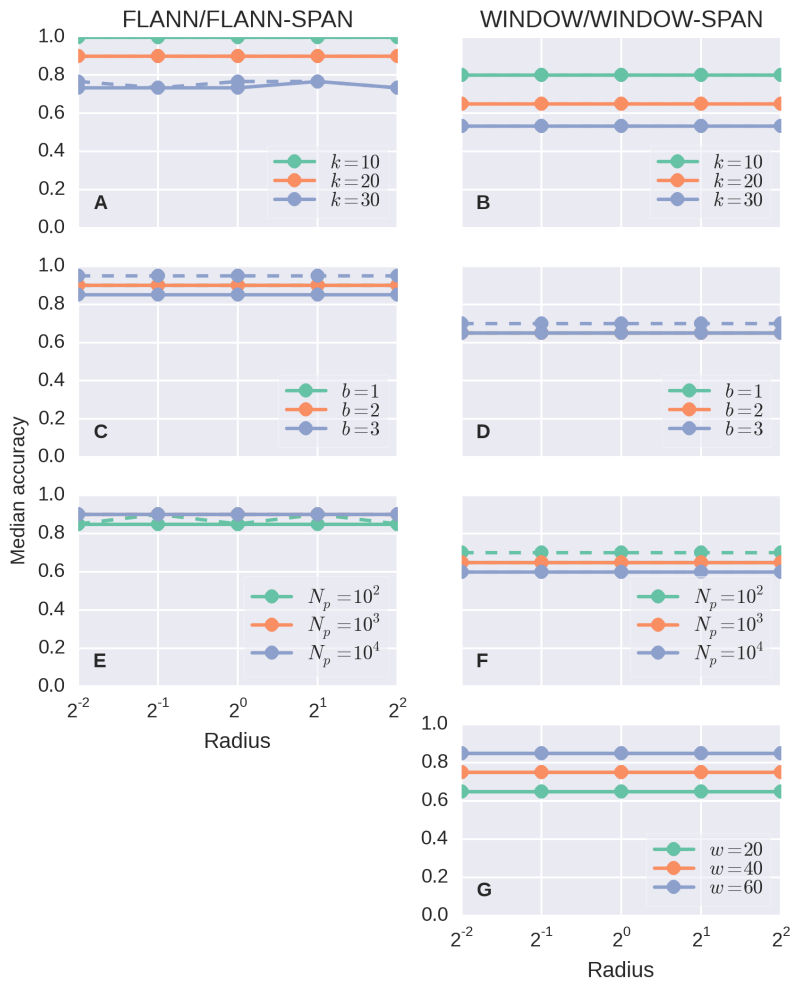

Figure 4: $\mathrm{kNN}$ accuracy in processing a hemispherical point cloud for different parameter values. Panels A, C and E employed either FLANN or FLANN-SPAN whilst panels B, D E and $\mathrm{G}$ employed either WINDOW or WINDOW-SPAN. Solid lines correspond to FLANN or WINDOW whereas broken lines are for FLANN-SPAN and WINDOW-SPAN. The varied parameters are (A-B) number of nearest neighbours $k$, (C-D) bits per dimension $b$, (E-F) number of points per partition $N_{\mathrm{p}}$ and (F) window look-back/look-ahead length $w$. If a parameter is not varying in a panel, value used is $b=2, N_{\mathrm{p}}=1000, k=20$ and $w=20$.

Except for a few particular instances as well, the (broken) lines for the spanning version of each workflow coincide with the nonspanning version, which also suggests that the added complication of spanning does not usually result to any significant improvement in the accuracy.

One can easily notice in Figure 4A that the median accuracy for $k=10$ is 1.0 which implies that as long as $k \leq 10$ then the selected nearest neighbours are likely to be all correct. As $k$ increases the accuracy decreases, which is expected since as more neighbours are required, the likelihood of one of these neighbours being outside the partition increases. Adding spanning mitigated this partition effect slightly as shown in Figure 4A but the line for $k=30$ (FLANN-SPAN) is too close and too intertwined (the accuracy at $r=2$ is worse than for FLANN) for us to make any stronger conclusions. The drop in the accuracy due to increasing $k>k_{\text {thres }}$ after some threshold $k_{\text {thres }}$ seems to be nonlinear since the change in median accuracy from $k=10$ to $k=20$ is 0.1 whilst from $k=20$ to $k=30$ is 0.17 .

Comparing the results with that for WINDOW and WINDOW-SPAN (Figure 4B), the difference in the accuracy is more apparent. Whilst for $k=10$, the median accuracy for FLANN and FLANN-SPAN is 1.0 , the median accuracy for WINDOW and WINDOW-SPAN is 0.8 which is closer to the median accuracy of 0.73 for $k=30$ (FLANN). Using spanning does not seem to have an effect on the median accuracy. As expected, the median accuracy decreases as 
$k$ increases but unlike in FLANN and FLANN-SPAN, the change is more linear with a drop of 0.15 from $k=10$ to $k=20$, and 0.12 from $k=20$ to $k=30$.

A smaller $b$ means more points per partition for a constant total number of points $N$ in the point cloud. However, the $N_{\mathrm{p}}$ is held constant so what we are observing in Figures $4 \mathrm{C}$ and $4 \mathrm{D}$ are the effect of the $Z$-order and partitioning on the accuracy. In Figure $4 \mathrm{C}$, the median precision for FLANN is 0.9 for $b=1$ and $b=2$ and 0.85 for $b=3$. The values are the same for $b=1$ and $b=2$ for FLANN-SPAN but jumps to 0.95 for $b=3$. It seems that for $b=3$, the partitions are too small that a significant number of nearest neighbours are in other partitions such that enabling spanning significantly improves and beats the median precision for smaller values of $b$.

The advantage of enabling spanning is also observed in Figure 4D albeit the best median precision of 0.7 is still less than the worse precision of 0.85 for FLANN. These results suggest that spanning can improve accuracy for some minimum value of $b$ although we cannot confidently state the value of that threshold at this point. These also support the possible use of spanning in working around issues related to partitioning.

When $N_{\mathrm{p}}$ is increased, the number of points near a partition boundary increases but so are the number of points that are inside and that have all their nearest neighbours within the partition. The rate of increase of the latter should be more than that of the former so the net effect would be that the accuracy tends to increase as $N_{\mathrm{p}}$ increases. This effect is somewhat observed in Figure 4E where the median accuracy for FLANN jumped from 0.85 for $N_{\mathrm{p}}=10^{2}$ to 0.9 for $N_{\mathrm{p}}=10^{3}$ and stayed there for $N_{\mathrm{p}}=10^{4}$. Spanning improved the median accuracy of FLANN-SPAN to 0.9 for $r=2^{-2}$ and $r=2^{2}$, which may be related to the area covered by the point cloud in corner partitions.

The expected effect of $N_{\mathrm{p}}$ is opposite to what is observed in Figure 4F. The median accuracy of WINDOW is 0.65 for both $N_{\mathrm{p}}=10^{2}$ and $N_{\mathrm{p}}=10^{3}$ but drops to 0.6 for $N_{\mathrm{p}}=10^{4}$. This unexpected result can be explained by recalling that WINDOW relies on sorting based on $Z$-order. As more points are added, the bias against nearest neighbours that are not along the traversal direction of the $Z$-order increases leading to a decrease in the accuracy. Enabling spanning improves the median accuracy to $N_{\mathrm{p}}=0.7$.

Not surprisingly, increasing $w$ improves accuracy as shown in Figure 4G. In this case, the median accuracy linearly increased from 0.65 for $w=20$ to 0.85 for $w=60$. Since $N_{\mathrm{p}}=1000$, this corresponds to a window size of $4.1 \%$ to $12.1 \%$ of the number of points in the partition. Spanning has no effect on the median accuracy.

\subsection{Triangular Wave Point Cloud}

We test the workflows on a non-smooth, triangular wave point cloud with $p$ peaks parallel to the $y$-axis and residing in the $+z$ half-space.

3.2.1 Spatial Accuracy The accuracy distribution of FLANN and FLANN-SPAN, and WINDOW and WINDOW-SPAN (Figure 5) for a triangular wave point cloud is similar within each pair. The triangular wave peaks are oriented vertically in the figure.

There are noticeable horizontal lines in Figures 5A and 5B, which are due to the lower accuracy of points near partition boundaries. There is only one visible vertical line and this corresponds to the
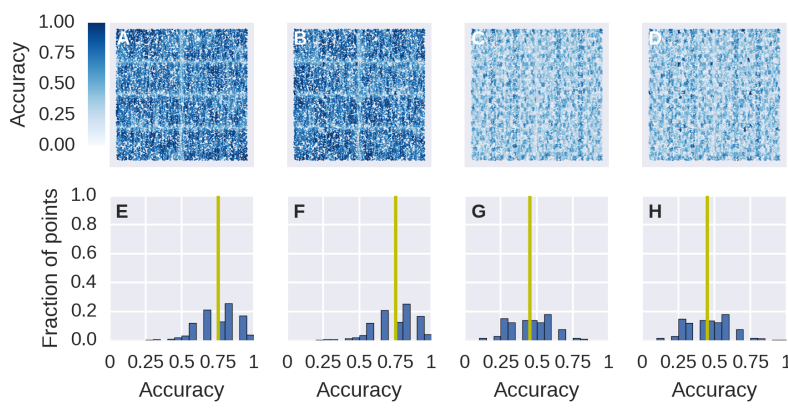

Figure 5: kNN accuracy in processing a triangular wave point cloud for a particular set of parameters for different workflows. In A-D, the points are projected onto the $x-y$ plane. The colour corresponds to the $\mathrm{kNN}$ accuracy, defined as the fraction of correctly selected $k$ nearest neighbours, at each point. In E-H, the distribution of the accuracy is shown with the yellow vertical line indicating the value of the median. The workflows are: (A,E) FLANN, (B,F) FLANN-SPAN, (C,G) WINDOW, (D,H) WINDOW-SPAN. The values of the parameters are bit per dimension $b=2$, number of points per partition $N_{\mathrm{p}}=1000$, number of peaks $p=3$, number of nearest neighbours $k=20$ and look-back/look-ahead length $w=20$.

only triangular wave peak that coincides with a partition boundary. Taken together, the position of the visible lines imply that points near partition boundaries are not guaranteed to have lower accuracy than inner points.

It is harder to identify any prominent features in Figures 5C and 5D but, upon closer inspection, a light vertical line can be made out at the middle, which again corresponds to the triangular wave peak that coincides with a partition boundary. Two dark lines at each side of the light vertical line can also be seen and these correspond to triangular wave peaks and troughs.

All workflows have lesser accuracy (Figure 5E-H) when the input is a triangular wave point cloud instead of a hemispherical point cloud. From a median accuracy of 0.9 for a hemispherical point cloud, the median accuracy of FLANN and FLANN-SPAN for a triangular wave point cloud is 0.75 . Similarly, the median accuracy of WINDOW and WINDOW-SPAN dropped from 0.65 to 0.45 . The peaks are less prominent and shifted to the left as well. The dip in the accuracy is expected because a nearest neighbour of a point may be on the other side of a peak or dip and this would be farther along in sequence of the $Z$-order.

3.2.2 Effect of Different Parameters on Accuracy There is a general trend of decreasing accuracy for increasing peaks (Figure 6), which is expected. FLANN and FLANN-SPAN are also more accurate than WINDOW and WINDOW-SPAN.

Unlike with hemispherical point cloud where $k \leq 10$ results to a median accuracy of 1.0 for FLANN and FLANN-SPAN in all values of $r$ considered, for triangular wave point clouds (Figure 6A), it is 1.0 only if $p \leq 2$. This can be extended to $p=3$ if spanning is enabled but if $p>3$, the median accuracy is constant at 0.9 . Enabling spanning also improves the median accuracy for $p=$ 3 when $k=20$. However, spanning results in lower median precision for $k=30$. Spanning has no effect on the accuracy of WINDOW and WINDOW-SPAN (Figure 6B).

The distance between the $k=10$ and $k=20$ curves is noticeably larger than that between the $k=20$ and $k=30$ curves for all workflows. The difference in distance suggests that accuracy is 

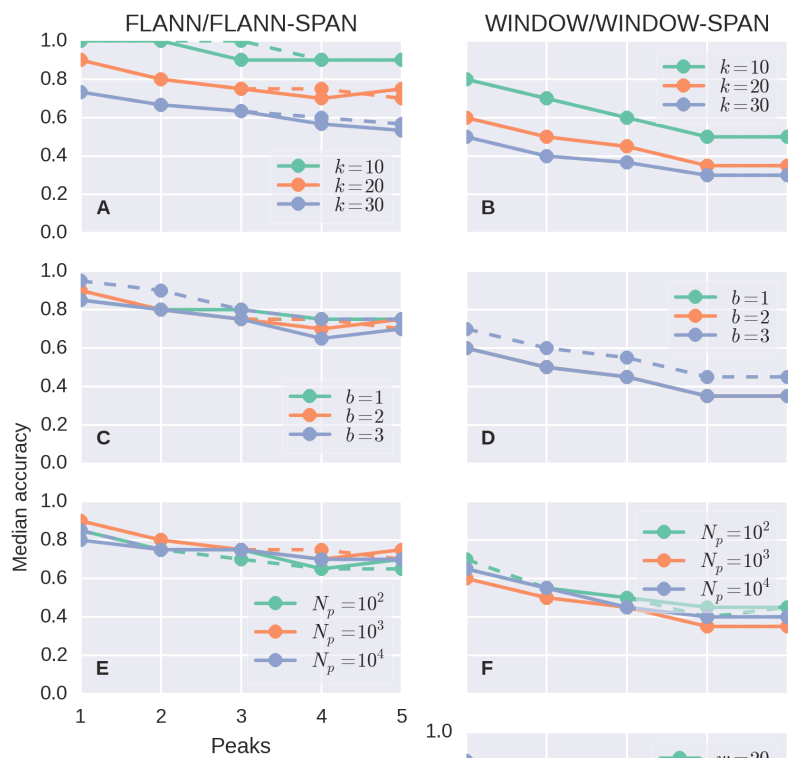

D

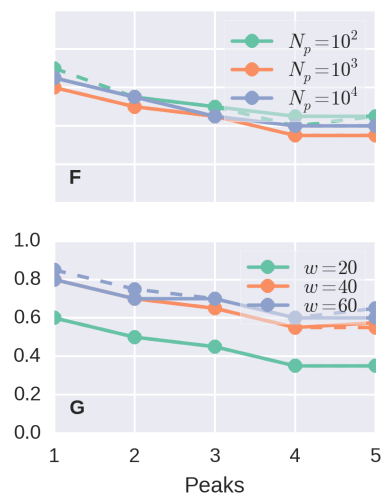

Figure 6: kNN accuracy in processing a triangular wave point cloud for different parameter values. Panels A, C and E employed either FLANN or FLANN-SPAN whilst panels B, D E and G employed either WINDOW or WINDOW-SPAN. Solid lines correspond to FLANN or WINDOW whereas broken lines are for FLANN-SPAN and WINDOW-SPAN. The varied parameters are (A-B) number of nearest neighbours $k$, (C-D) bits per dimension $b$, (E-F) number of points per partition $N_{\mathrm{p}}$ and (F) window look-back/look-ahead length $w$. If a parameter is not varying in a panel, value used is $b=2, N_{\mathrm{p}}=1000, k=20$ and $w=20$.

more sensitive between $k=10$ and $k=20$ than between $k=20$ and $k=30$.

The curves in Figure 6C are overlapping so it is difficult to ascertain the effect of $b$ on the median accuracy. However, $b=3$ tends to have the smallest median accuracy for FLANN but enabling spanning makes the workflow the most accurate among the curves in Figures 6C and D. This is more acutely seen in Figure 6D wherein the curve for $b=3$ (WINDOW-SPAN) is clearly above the other curves, which are coinciding. The same behaviour of $b=3$ (WINDOW-SPAN) is also seen for a hemispherical point cloud and the same explanation holds.

The curves are overlapping in Figures $6 \mathrm{E}$ and $\mathrm{F}$ so it is difficult to issue a strong statement on the effect of $N_{\mathrm{p}}$ on the median accuracy of different workflows. The observation on hemispherical point clouds that median accuracy tends to increase with $N_{\mathrm{p}}$ for FLANN and FLANN-SPAN but tends to decrease for WINDOW and WINDOW-SPAN no longer holds.

There is a general trend of increasing median accuracy for increasing $w$ as shown in Figure 6G. However, the trend is less defined than in hemispherical point cloud because of overlapping curves. There is an improvement in the median accuracy in some instances if spanning is enabled.

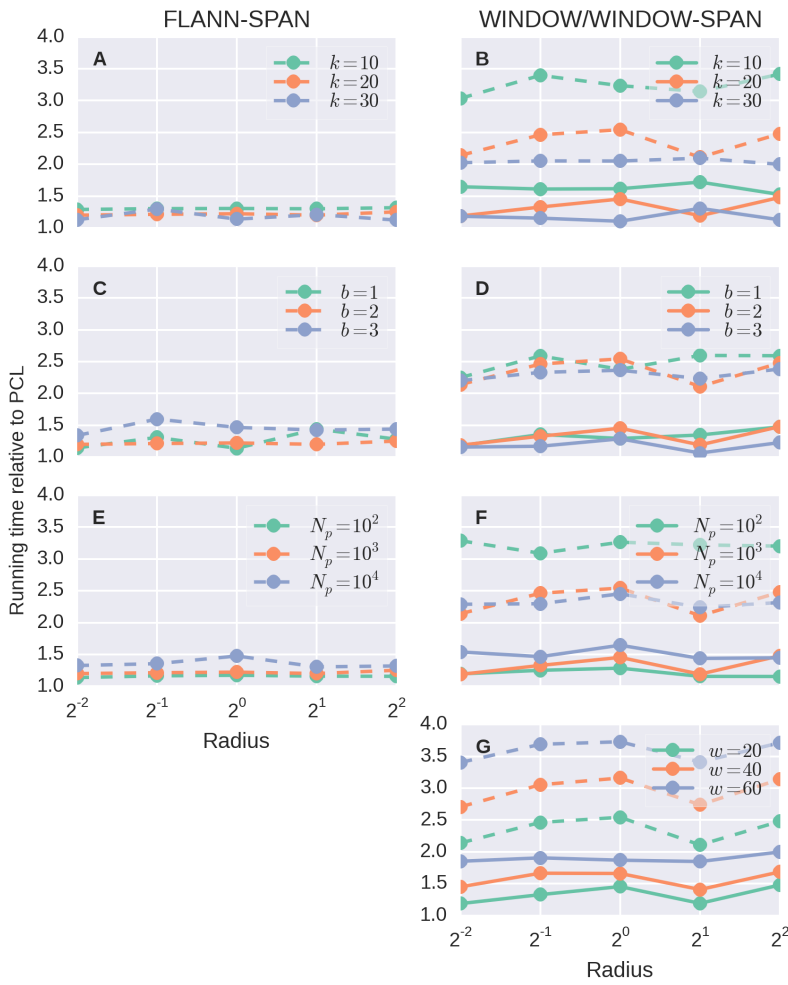

Figure 7: Relative running times of kNN for processing a hemispherical point cloud. Relative running time is computed as the ratio between the running time of each workflow and the running time of FLANN and is based on the mean of five trials. Panels A, C and E are for FLANN-SPAN only whilst panels B, D E and G employed either WINDOW or WINDOW-SPAN. Solid lines correspond to WINDOW whereas broken lines are for FLANN-SPAN and WINDOW-SPAN. The varied parameters are (A-B) number of nearest neighbours $k$, (C-D) bits per dimension $b$, (E-F) number of points per partition $N_{\mathrm{p}}$ and (F) window look-back/look-ahead length $w$. If a parameter is not varying in a panel, the value used is $b=2, N_{\mathrm{p}}=1000, k=20$ and $w=20$.

\subsection{Running Time}

The running time for both hemispherical and triangular wave point clouds are similar so we only analyse that of the hemispherical point cloud.

The fastest workflow is FLANN as shown in Figure 7 where all relative running times are greater than 1.0. Spanning workflows are also slower than their corresponding non-spanning workflows because these involve an extra shuffle.

The relative running times of FLANN-SPAN with respect to $r$ (Figure 7A) is almost constant for $k=10$ and $k=20$ with the former having consistently larger relative running times (1.29 to 1.32) than the latter (1.20 to 1.25$)$. The relative running time for $k=30$ is more erratic with it being almost the same as in $k=10$ for $r=2^{-1}$ and as in $k=20$ for $r=2^{1}$. The apparent decreasing relative running time as $k$ increases suggest that the overhead of spanning decreases as $k$ increases.

The somewhat erratic behaviour of the relative running time as $r$ increases is also observed (Figure 7B) in WINDOW and WINDOWSPAN. However, there is a general trend that relative running time increases as $k$. Although WINDOW is slower than FLANN-SPAN, 
the relative running time of WINDOW does not exceed 1.7 compared to that of WINDOW-SPAN which is at least 2.0. In general, the relative running time of WINDOW-SPAN is about twice that of WINDOW.

The relationship between relative running time and $r$ remains indefinite when $b$ (Figure 7C) is varied. The effect of $b$ itself is also irregular with the line for $b=1$ criss-crossing the line for $b=2$. However, the line for $b=3$ which is not too surprising because larger $b$ implies more partitions to shuffle.

Enabling spanning on WINDOW (Figure 7D) would result to an around $75 \%$ increase in relative running time for varying $b$. The lines for different $b$ are too intertwined to conclude as to the effect of $r$ on the relative running time.

There is an unexplained dip at $r=2^{1}$ that can be seen in Figure 7E-G. The maximum relative running time is at $r=2^{0}$ as well. Although there is a superlinear increase in the relative running time (Figure 7E) as $N_{\mathrm{p}}$ is increased, the maximum relative running time remains below 1.5. Enabling spanning on WINDOW (Figure 7F) doubles the relative running time and can exceed three times the running time of FLANN for $N_{\mathrm{p}}=10^{4}$. Increasing $w$ (Figure $7 \mathrm{G}$ ) results to a larger relative running time and enabling spanning doubles running time.

\section{CONCLUSION}

The increasing volume of point clouds increases the need for processing them in parallel. This would require breaking them down into partitions and distributing across multiple machines. Data locality is important here, which is the concept that data must be on the processing machine as much as possible because network transfer would be very slow.

We proposed a $Z$-order-based partitioning scheme and implemented it on Apache Spark. We showed that it is an effective method and a convenient one as well because third-party libraries and code may still be used. Enabling spanning may not be too useful as well as the improvement in median accuracy is minimal.

We have only investigated the accuracy of kNN selection but the accuracy of other nearest-neighbourhood-based operations may be investigated as well. For example, extracting normals from point clouds using PCA may prove to be more robust to the effects of partitioning.

An advantage of $Z$-order partitioning is that the level of detail i.e., partition size, can be easily adjusted. We intend to implement an adaptive $Z$-order indexing such that partitions can be automatically adjusted to have a more constant number of points per partition.

\section{ACKNOWLEDGEMENTS}

We would like to acknowledge that this work is in part supported by EU grant FP7-ICT-2011-318787 (IQmulus).

\section{REFERENCES}

Connor, M. and Kumar, P., 2010. Fast construction of k-nearest neighbor graphs for point clouds. IEEE Transactions on Visualization and Computer Graphics 16(4), pp. 599-608.
Eldawy, A., Alarabi, L. and Mokbel, M. F., 2015. Spatial Partitioning Techniques in SpatialHadoop. Proc. VLDB Endow. 8(12), pp. 1602-1605.

Lawder, J. K. and King, P. J. H., 2000. Using Space-Filling Curves for Multi-dimensional Indexing. In: B. Lings and K. Jeffery (eds), Advances in Databases, Lecture Notes in Computer Science, Springer Berlin Heidelberg, pp. 20-35.

Lu, W., Shen, Y., Chen, S. and Ooi, B. C., 2012. Efficient Processing of K Nearest Neighbor Joins Using MapReduce. Proc. VLDB Endow. 5(10), pp. 1016-1027.

Nishimura, S., Das, S., Agrawal, D. and Abbadi, A. E., 2012. MD-HBase: design and implementation of an elastic data infrastructure for cloud-scale location services. Distributed and Parallel Databases 31(2), pp. 289-319.

Orenstein, J. A. and Manola, F. A., 1988. PROBE spatial data modeling and query processing in an image database application. IEEE Transactions on Software Engineering 14(5), pp. 611-629.

Swart, L. T., 2010. How the Up-to-date Height Model of the Netherlands (AHN) became a massive point data cloud. NCG KNAW.

Wei, L.-Y., Hsu, Y.-T., Peng, W.-C. and Lee, W.-C., 2014. Indexing spatial data in cloud data managements. Pervasive and Mobile Computing 15, pp. 48-61.

You, S., Zhang, J. and Gruenwald, L., 2015. Large-scale spatial join query processing in Cloud. In: 2015 31st IEEE International Conference on Data Engineering Workshops (ICDEW), pp. 3441.

Zhang, C., Li, F. and Jestes, J., 2012. Efficient Parallel kNN Joins for Large Data in MapReduce. In: Proceedings of the 15th International Conference on Extending Database Technology, EDBT '12, ACM, New York, NY, USA, pp. 38-49. 